\title{
Perfect Versus Imperfect Monitoring in Multi-agent Opportunistic Channel Access
}

\author{
Ji Wang ${ }^{\dagger}$, Irene Macaluso*, Luiz A. DaSilva*† \\ *CTVR Telecommunications Research Center, Trinity College Dublin, Ireland \\ ${ }^{\dagger}$ Department of Electrical and Computer Engineering, Virginia Tech, U.S.A. \\ Email: traceyw@vt.edu, macalusi,dasilval@tcd.ie
}

\begin{abstract}
This paper investigates the impact of imperfect information on the performance of secondary users ( $\mathrm{SU}$ ) attempting to opportunistically exploit spectrum resources in a distributed manner. We design two channel selection strategies that leverage different levels of information about past channel activity. In the case of perfect monitoring we assume each SU is able to distinguish signals transmitted by a primary user (PU) from signals transmitted by an SU and collision events. In the case of imperfect monitoring we assume SUs to be unable of discriminating between SU or PU activity in the channel. We formally prove that both strategies converge to a static orthogonal channel allocation. Our simulation results show that the impact of imperfect information is to reduce the rate of convergence; the performance loss depends on the level of PU activity and on the amount of competition between SUs.
\end{abstract}

Index Terms-Dynamic spectrum access, opportunistic channel access, imperfect monitoring, cognitive radio network, SU channel selection strategy.

\section{INTRODUCTION}

This paper investigates the impact of imperfect information on the performance of secondary users (SU) attempting to opportunistically exploit spectrum resources in a distributed manner. We consider a multi-channel band where SUs can be active on a non-interfering basis, such as in authorized shared access. The objective of a strategy designed for opportunistic spectrum access in the case of multiple SUs is twofold: each SU should try to exploit the channel that is most likely to be free of primary users (PU); and each SU should minimize the likelihood of collision with other SUs. This work addresses the question of how the quality of the information about past activity by the PU or SUs in each channel affects the performance of SUs pursuing these objectives.

We consider two cases: under perfect monitoring, we assume each SU is able to distinguish signals transmitted by a PU from signals transmitted by another SU, as well as from collision events; under imperfect monitoring, we assume SUs to be unable of discriminating between SU or PU activity in the channel. Perfect monitoring implies the adoption of more sophisticated sensing techniques, which in turn translates into additional costs in terms of computational resources. Imperfect monitoring relies on a less informative channel state, which

This work was pursued under the Wireless Innovation between Finland and the US (WiFiUS) program, funded by the National Science Foundation under grant no. 1265280 . It was also partially funded by the Science Foundation can affect the SU's ability to learn from past observations and, as a consequence, deteriorate the system performance.

In order to focus our attention on the impact of imperfect information on the performance of channel selection strategies, we consider a simple but widely adopted model of PU channel occupancy. In particular, we assume that PU activity is independent and identically distributed across multiple channels and time slots. We devise a strategy for SU decisions under perfect monitoring and a strategy under imperfect monitoring, and we compare their performance. Our theoretical findings show that, under the i.i.d. channel model and assuming the number of SUs is less than the number of channels in the system, both perfect and imperfect monitoring strategies converge to a static orthogonal allocation of the channels across SUs. However, our results show that under imperfect monitoring the ambiguity about the type of activity observed in the channels reduces the rate of convergence. The degradation in convergence time depends on the level of PU activity and on the amount of competition between SUs.

The research presented in this paper can be situated within the broader class of opportunistic channel access strategy design in distributed cognitive radio networks. The primary contributions of this paper are to:

- Design channel selection strategies to be adopted by autonomous SUs, for the cases of perfect and imperfect monitoring of past channel usage.

- Analyze the impact of imperfect information regarding past channel occupancy on the ability of SUs to dynamically exploit the band.

We begin with a brief discussion of the state of the literature on opportunistic channel selection strategy design in Section II. Section III introduces the system model and related notation. The strategy design is described in Section IV with both perfect and imperfect monitoring. The theoretical performance analysis of the designed strategies is discussed in Section V. Section VI presents the simulation results. We summarize our conclusions and point towards directions for future work in Section VII.

\section{RELATED WORK}

Distributed opportunistic channel selection is a major research topic in cognitive radio networks [1]. Such channel selection strategies can be categorized into parallel sensing and sequential sensing (e.g., [2], [3]). The channel access strategy design in our paper is based on the parallel sensing technique. 
Most existing parallel sensing channel selection models assume the SUs to be capable of distinguishing whether, at any given time, the channels have been occupied by the PU or by another SU, which can be achieved either through cooperative spectrum sensing [4] [5] or advanced sensing techniques [6] [7]. [8]-[10] provide repeated game frameworks to model the dynamic spectrum access among non-cooperative SUs. However, these models also require the SU to distinguish between SUs' and PUs' transmissions.

Some recent research papers mention the "imperfect monitoring" concept. [11] and [12] consider the imperfect monitoring based on an underlay framework where PUs impose interference temperature constraints. In their model imperfect monitoring refers to erroneous measurements of the interference temperature. The imperfect monitoring in [13] [14] [15] is about the sensing error in SUs' observed information. The imperfect monitoring information in our paper refers to the inability of discriminating between SU or PU activity in a channel.

\section{System Model AND Notation}

We consider a time-slotted cognitive radio system consisting of $M$ homogeneous channels and $N$ SUs. At the beginning of a time slot, each SU chooses exactly one channel to sense and transfer data on if no PU is sensed to be using that channel. If a PU is found to be active in the selected channel, the SU will wait during that time slot without transmitting or further sensing other channels. If more than one SUs sense the same channel and transmit data simultaneously, a collision will happen and none of them will deliver data successfully. Thus, a successful transmission occurs on a given channel if only one SU has chosen this channel and no PU is active on this channel. At the end of each time slot, an SU gets feedback on whether its transmission was successful (for instance, in the form of an ACK).

The key decision for each SU to make in this channel selection scheme is which channel to exploit for each time slot to maximize the long term payoff, i.e., which channel is most likely to be free of PUs and of conflicts with the other SUs. This decision can be made based on previous channel occupancy history. We explore two scenarios: (i) the decision is made autonomously by each SU with perfect monitoring of the prior history of channel utilization by PUs and SUs; and (ii) the decision is made autonomously by each SU with imperfect monitoring of the prior history. We further describe these two scenarios in the following paragraphs.

The channel occupancy information in time slot $t$ can be represented in an $M$-dimensional vector $\mathbf{y}(\mathbf{t})=$ $\left(y_{1}(t), y_{2}(t), \ldots, y_{M}(t)\right)^{T}$. The payoff of SU $i^{\prime} s$ attempt to transmit in slot $t$ can be represented as $u_{i}^{t} \in\{0,1\} . u_{i}^{t}=0$ represents a transmission failure due to the selected channel being occupied by a PU or due to collision with another SU. $u_{i}^{t}=1$ represents success. At the beginning of time slot $t, \mathrm{SU}$ $i$ makes channel selection decision $a_{i}^{t}$ based on the observed channel occupancy history $\{\mathbf{y}(k)\}$ and its own past payoff $u_{i}^{k}$, for $k \in\{1, \ldots, t-1\}$. The optimization goal of channel selection strategy design is to maximize the expected long term individual utility $E\left[u_{i}\right]=(1-\delta) \sum_{k=0}^{\infty} \delta^{k} E\left[u_{i}^{k}\right]$, where $\delta \in(0,1)$ is a discount factor. This factor can be understood as an indication of how much SUs care about future payoffs. The more patient the SU is, the closer this value is to one.

We describe two scenarios in this paper, which differ in terms of the responsibility for the channel selection decision and the availability of past channel information. Firstly, we consider a distributed cognitive radio system where SUs act autonomously without exchanging information with each other. At the end of time slot $t$, we assume SUs are capable of sensing all $M$ channels and classifying the signals they encounter, distinguishing signals transmitted by a PU from signals transmitted by an SU and collision events. The resulting information is denoted as $y_{j}^{p m}(t) \in\{0,1,2,3\}$, where $y_{j}^{p m}=0$ represents the event that channel $j$ was idle, $y_{j}^{p m}=1$ represents that channel $j$ was occupied by a PU, $y_{j}^{p m}=2$ represents that channel $j$ was occupied by one $\mathrm{SU}$, and $y_{j}^{p m}=3$ represents the occurrence of a collision between more than one SUs. The superscript $\mathrm{pm}$ denotes perfect monitoring, as the $\mathrm{SU}$ is able to determine what type of user (another SU or a PU) was active in that channel.

Secondly, we consider an autonomous cognitive radio system under the imperfect monitoring scenario. The only difference between the imperfect monitoring and the perfect monitoring scenarios lies in the observed channel occupancy signal. Under imperfect monitoring, we assume SUs to be capable of sensing all $M$ channels at the end of time slot $t$, using a simple sensing technique such as energy detection. SUs are, however, incapable of classifying the observed signals and distinguishing between SU and PU activity in the channel. The occupancy signal for channel $j$ is denoted as $y_{j}^{i m}(t) \in\{0,1\}$, where $y_{j}^{i m}=0$ represents that channel $j$ was not occupied and $y_{j}^{i m}=1$ represents that channel $j$ was occupied (by a $\mathrm{PU}$, an SU, or due to a collision between multiple SUs). The superscript $i m$ denotes imperfect monitoring.

In this paper, we consider the PU activity to be independent and identically distributed (i.i.d.) across the channels as well as the time slots. We assume all channels have the same duty cycle $(D C)$. We further assume that $M>N+1$.

\section{Strategy Design}

In this section, we present the channel selection strategies under perfect and imperfect information monitoring scenarios. Under a homogeneous channel model, where all channels are equally likely to be occupied by a PU, the goal of a channel selection strategy is to avoid collision among SUs. Since we assume the number of channels is greater than the number of SUs, our goal is to design a channel selection strategy such that SUs autonomously arrive at orthogonal channel allocations.

A common feature of the two strategies described in the remainder of this section is the behavior of SUs after a successful transmission. In particular, under both perfect and imperfect monitoring scenario, an SU that has successfully transmitted data on a channel will continue to exploit the same channel in subsequent time slots. Such an SU is henceforth referred to as a static SU. 
Perfect Monitoring Scenario: The strategy under perfect monitoring starts with each SU randomly exploring one channel to sense. As mentioned before, if an SU successfully transmits on a channel, it continues to exploit the same channel in subsequent time slots, i.e. it becomes a static SU. Otherwise, from the next time slot, a non-static SU randomly selects one channel among the channels that were not successfully used by any $\mathrm{SU}$ in the previous time slots. In the process, each SU updates a length- $M$ binary vector $C=\left(c_{1}, \ldots, c_{M}\right): c_{j}$ is set to 1 when channel $j$ is observed used by an SU. Algorithm 1 describes the channel selection process under the perfect monitoring scenario.

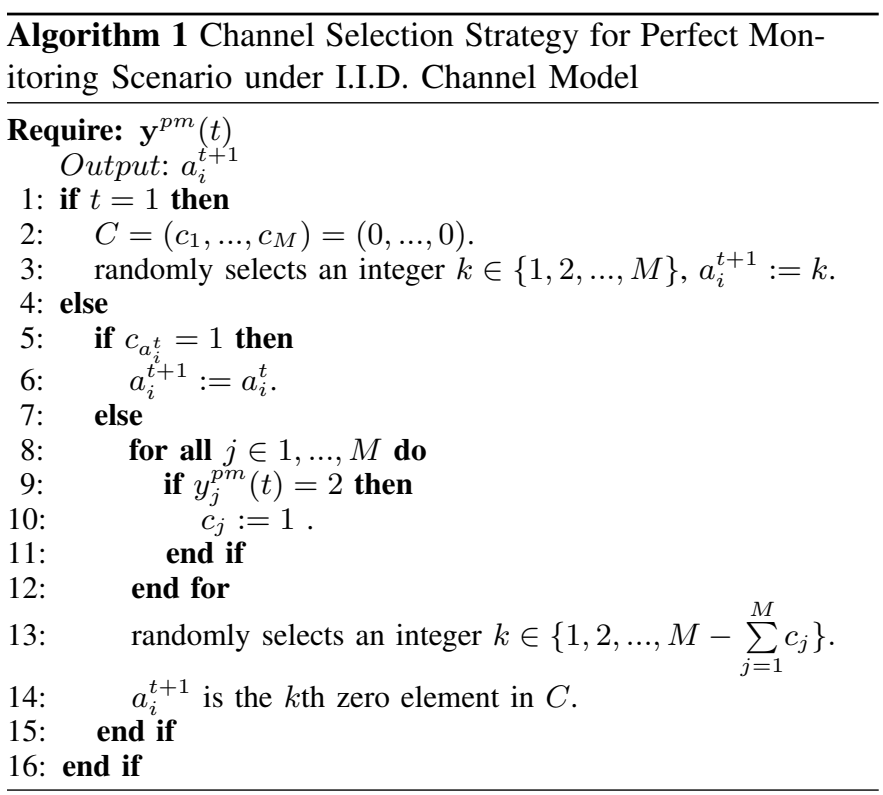

Imperfect Monitoring Scenario: For the strategy under imperfect monitoring, each SU randomly senses one channel in the first time slot. Whenever an SU's transmission on a channel is successful, the SU becomes static, i.e., it continues to exploit the same channel in subsequent time slots; otherwise, it randomly selects among the channels observed idle in the last time slot. The rationale behind this design is to avoid non-static SUs from colliding with static SUs. If all channels were observed occupied in the past time slot, a non-static SU selects the same channel as it did in the previous time slot. Algorithm 2 describes this process at a generic time $t$.

\section{Strategy Analysis}

In this section we conduct a theoretical study of the convergence properties of the two strategies described above. According to the channel selection strategies defined in the previous section, if at any time slot all SUs are static, they will remain static for all subsequent time slots and they will not collide with each other. In other words, when all SUs are static the channel selection strategies have converged to a static orthogonal channel allocation. The expected long-term payoff of an $\mathrm{SU} i$ is lower bounded by:

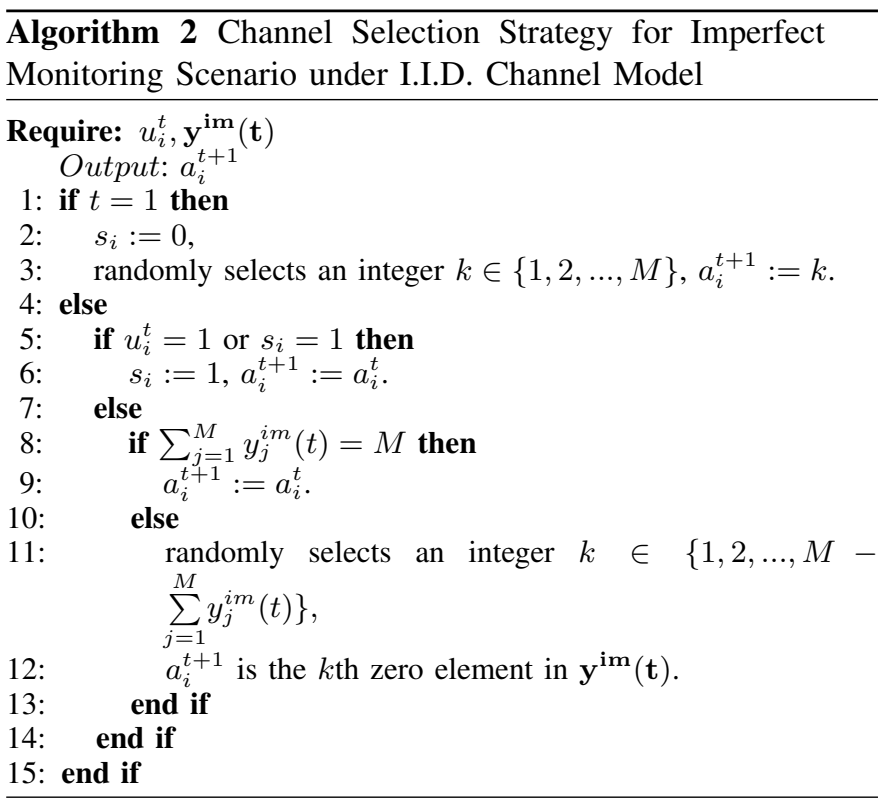

$$
\begin{aligned}
E\left[u^{i}\right] & >(1-\delta)\left(\sum_{t=0}^{k} u^{t}(t) \delta^{t}+\sum_{t=k+1}^{\infty}(1-D C) \delta^{t}\right) \\
& \geq(1-D C) \delta^{k+1},
\end{aligned}
$$

where $k$ is the number of time slots before SU $i$ becomes static.

Inequality 1 indicates that the faster a strategy converges the higher the lower bound of the expected long-term individual payoff will be. It should be noted that, under the assumption of i.i.d. homogeneous PU activity, the expected long-term individual payoff is at most equal to $1-D C$.

\section{A. Perfect Monitoring Strategy Convergence Analysis}

We start by analyzing the convergence of the channel selection strategy under perfect monitoring.

Let us denote by $S_{t}^{p m}$ the number of static secondary users before time slot $t+1$. According to the strategy described in Section IV, $S_{1}^{p m} \leq S_{2}^{p m} \leq \ldots \leq S_{t}^{p m} \leq N$. Also, at time slot $t+1$ each non-static SUs randomly selects a channel among the $M-S_{t}^{p m}$ channels that have never been successfully occupied by other SUs. Hence, the probability of each nonstatic SUs successfully transmitting on a channel at time $t+1$, i.e. the probability of becoming a static SU, only depends on the current number of static SUs, on the number of channels $M$ and on the $D C$. This means that the sequence of random variables $S_{1}^{p m}, S_{2}^{p m}, \ldots, S_{t}^{p m}$ is a Markov chain $T$ with state space $\{0,1, \ldots, N\}$ and transition probability matrix $T_{i j}$ as shown in Figure 1.

We denote the conditional probability that $j$ static SUs are static before $t+1$ given $i$ SUs were static before $t$ as $T_{i j}^{p m}$, i.e., $T_{i j}^{p m}=P\left(S_{t}^{p m}=j \mid S_{t-1}^{p m}=i\right)$. We denote by $Y_{t}^{p m}$ the number of SUs who become static in time slot $t$. Therefore, $T_{i j}^{p m}=P\left(Y_{t}^{p m}=j-i \mid S_{t-1}^{p m}=i\right)$. Therefore, $T_{i j}^{p m}=0$ for $0 \leq j<i \leq N$. Also, $T_{N, N}^{p m}=1$. When $i$ SUs are already static before $t$, the remaining $N-i$ non-static SUs 
randomly select among the $M-i$ channels that have never been successfully occupied by static SUs. Hence, given the total number of static SUs before $t$, the number of SUs that become static in the $t$ is a binomial random variable with probability mass function:

$$
\begin{aligned}
T_{i j}^{p m} & =P\left(S_{t}^{p m}=j \mid S_{t-1}^{p m}=i\right) \\
& =P\left(Y_{t}^{p m}=j-i \mid S_{t-1}^{p m}=i\right) \\
& =\left(\begin{array}{c}
N-i \\
j-i
\end{array}\right)\left(P_{t}^{p m}\right)^{j-i}\left(1-P_{t}^{p m}\right)^{N-j},
\end{aligned}
$$

for $0 \leq i \leq j \leq N$ and $i \neq N$, where $P_{t}^{p m}$ is the probability for a non-static SU to successfully transmit data in time slot $t$, and can be expressed as:

$$
P_{t}^{p m}=(1-D C)\left(1-\frac{1}{M-i}\right)^{N-i-1}
$$

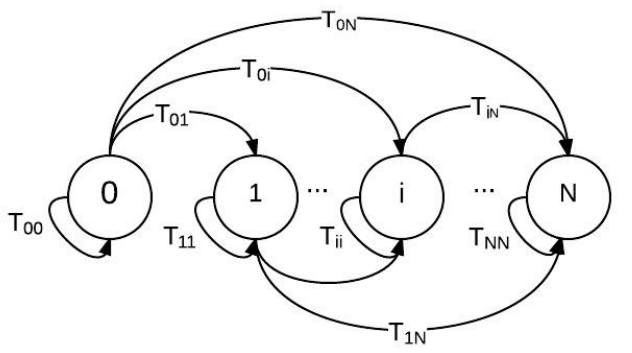

Figure 1: Markov Chain T

In the remainder of this section we will show that $T$ is an absorbing Markov chain; we will use this result to prove convergence of the strategy under perfect monitoring and express the convergence time as a function of the transition probability matrix $\left[T_{i j}\right]$.

Definition 1. [16] A state of a Markov chain is called absorbing if it is impossible to leave it. A Markov chain is absorbing if it has at least one absorbing state, and if from every state it is possible to go to an absorbing state (not necessarily in one step).

Lemma 2. $T$ is an absorbing Markov chain.

Proof: Since $T_{N, N}^{p m}=1, N$ is an absorbing state. For $i \neq$ $N$, we have $T_{i N}^{p m}=\left(P_{t}^{p m}\right)^{N-i}$, where $P_{t}^{p m}=(1-D C)(1-$ $\left.\frac{1}{M-i}\right)^{N-i-1}>0$. Therefore $T_{i N}^{p m}>0$. Hence, according to definition $1 T$ is an absorbing Markov chain.

Lemma 3. $N$ is the unique absorbing state of Markov chain $T$.

Proof: Suppose there exists a state $k$ other than $N$ such that $k$ is an absorbing state of $T$, i.e. $k \neq N, T_{k, k}^{p m}=1$. During the deduction of lemma 2, we have $T_{k, N}^{p m}>0$. Therefore, $T_{k, k}^{p m}=1-\sum_{j=0}^{N} T_{k, j}^{p m} \leq 1-T_{k, N}^{p m}<1$, which is a contradiction to the assumption $T_{k, k}^{p m}=1$. Therefore, no such state $k$ exists.

We have proved that $T$ is an absorbing Markov chain with a unique absorbing state $N$. Hence according to [16], the probability that after $t$ time slots all $N$ SUs become static under the strategy designed for the perfect monitoring scenario approaches one as $t$ goes to infinity.

Definition 4. [17] For an absorbing Markov chain $P$ with $r$ absorbing states, the transition matrix can be written as $\left(\begin{array}{ll}Q & R \\ 0 & I_{r}\end{array}\right)$, where $I_{r}$ is an $r$ by $r$ identity matrix. The matrix $N=(I-Q)^{-1}$ is called the fundamental matrix for $P$. The entry $n_{i j}$ of $N$ gives the expected number of times that the process is in the transient state $s_{j}$ if it is started in the transient state $s_{i}$.

Theorem 5. [17] Let $t_{i}$ be the expected number of steps before the chain is absorbed, given that the chain starts in state $s_{i}$, and let $\mathrm{t}$ be the column vector whose ith entry is $t_{i}$. Then $t=N c$, where $c$ is a column vector all of whose entries are equal to 1 .

Corollary 6. The expected convergence time for the strategy under perfect monitoring scenario is the summation of the first row of $N$, where $N=\left(\begin{array}{cccc}1 & 0 & \cdots & 0 \\ 0 & 1 & \cdots & 0 \\ \vdots & \vdots & \ddots & \vdots \\ 0 & 0 & \cdots & 1\end{array}\right)-$ $\left.\left(\begin{array}{cccc}T_{0,0}^{p m} & T_{0,1}^{p m} & \cdots & T_{0, N-1}^{p m} \\ 0 & T_{1,1}^{p m} & \cdots & T_{1, N-1}^{p m} \\ \vdots & \vdots & \ddots & \vdots \\ 0 & 0 & \cdots & T_{N-1, N-1}^{p m}\end{array}\right)\right)^{-1}$

Proof: According to Theorem (4) the transition matrix

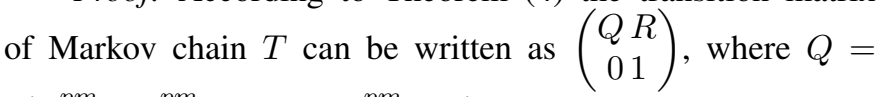
$\left(\begin{array}{cccc}T_{0,0}^{p m} & T_{0,1}^{p m} & \cdots & T_{0, N-1}^{p m} \\ 0 & T_{1,1}^{p m} & \cdots & T_{1, N-1}^{p m} \\ \vdots & \vdots & \ddots & \vdots \\ 0 & 0 & \cdots & T_{N-1, N-1}^{p m}\end{array}\right)$

By Theorem 5, the expected number of time slots it takes for the perfect monitoring strategy to converge, is the first element of the column vector $(I-Q)^{-1} c$ where $c$ is a $N$ by 1 column vector with all entries are equal to 1 .

\section{B. Imperfect Monitoring Strategy Convergence Analysis}

In this section, we prove the convergence of the strategy under imperfect monitoring scenario. We denote by $S_{t}^{i m}$ the total number of static SUs before time slot $t+1$ for the strategy under imperfect monitoring scenario, $S_{t}^{i m} \in\{1, \ldots, N\}$. Therefore $S_{t+1}^{i m} \geq S_{t}^{i m}$ for $t>0$.

Claim 7. If the channel selection strategy under imperfect monitoring does not converge, then $\exists t_{1}>0$ s.t. $P\left(S_{t_{1}+1}^{i m}=\right.$ $\left.k \mid S_{t_{1}}^{i m}=k\right) \equiv 1$ for $k \in\{0,1, \ldots N-1\}$.

Proof: Assume the strategy does not converge, i.e., $S_{t}^{i m} \leq$ $N-1$ for $\forall t>0$. Let us suppose that for $\forall t>0, P\left(S_{t+1}^{i m}=\right.$ $\left.k \mid S_{t}^{i m}=k\right)<1$, with $k \in\{0,1, \ldots N-1\}$. 
As $S_{t}^{i m} \leq S_{t+1}^{i m} \leq N-1$, we have $P\left(S_{t+1}^{i m}<k \mid S_{t}^{i m}=\right.$ $k) \equiv 0$. Therefore,

$$
P\left(S_{t+1}^{i m}>k \mid S_{t}^{i m}=k\right)=1-P\left(S_{t+1}^{i m}=k \mid S_{t}^{i m}=k\right)>0 .
$$

As $S_{t+1}^{i m}$ is a discrete random variable in time slot $t+1$ with $S_{t+1}^{i m} \in\{1,2, \ldots N-1\}$, (4) implies that there exists a positive integer $k_{1}$ such that

$$
P\left(S_{t+1}^{i m}=k+k_{1} \mid S_{t}^{i m}=k\right)>0 .
$$

If $S_{t+1}^{i m}=k+k_{1}=N$, it contradicts the assumption that $S_{t}^{i m} \leq N-1$ for $\forall t>0$. Otherwise, by applying a similar argument we can find a positive integer $k_{2}$ such that $P\left(S_{t+2}^{i m}=k+k_{1}+k_{2} \mid S_{t+1}^{i m}=k+k_{1}\right)>0$. Since $k_{1}, k_{2}, \ldots$ are positive integers, this process must stop within $N$ steps before it contradicts the assumption $S_{t}^{i m} \leq N-1$ for $\forall t>0$.

Theorem 8. The probability that $N$ SUs are static under imperfect monitoring approaches 1 as time goes to infinity.

Proof: Assume the imperfect monitoring strategy does not converge. By claim (7), $\exists t_{1}>0$ s.t. $P\left(S_{t_{1}}^{i m}=k \mid S_{t_{1}-1}^{i m}=\right.$ $k) \equiv 1$ for $k \in\{0,1, \ldots N-1\}$.

We denote by $Y_{t}^{i m}$ the number of non-static SUs that become static at time $t$. Therefore,

$$
P\left(S_{t_{1}}^{i m}=k \mid S_{t_{1}-1}^{i m}=k\right)=P\left(Y_{t_{1}}^{i m}=0 \mid S_{t_{1}-1}^{i m}=k\right) \equiv 1 .
$$

We further denote by $V_{t}^{i m}$ the number of idle channels in time slot $t$. Then, (6) can be expressed as:

$$
\begin{aligned}
1 & \equiv P\left(Y_{t_{1}}^{i m}=0 \mid S_{t_{1}-1}^{i m}=k\right) \\
& =\sum_{j=0}^{M-S_{t_{1}}^{i m}+1} P\left(V_{t_{1}-1}^{i m}=j\right) P\left(Y_{t_{1}}^{i m}=0 \mid S_{t_{1}-1}^{i m}=k, V_{t_{1}-1}^{i m}=j\right)
\end{aligned}
$$

which implies that when $P\left(V_{t_{1}-1}^{i m}=j\right)>0, P\left(Y_{t_{1}}^{i m}=\right.$ $\left.0 \mid S_{t_{1}-1}^{i m}=k, V_{t_{1}-1}^{i m}=j\right)=1$. We will show that this results in a contradiction since $P\left(V_{t_{1}-1}^{i m}=2\right)>0$ and $P\left(Y_{t_{1}}^{i m}=\right.$ $\left.0 \mid S_{t_{1}-1}^{i m}=k, V_{t_{1}-1}^{i m}=2\right)<1$ for $k=\{0,1, . . N\}$.

We denote by $L_{t}$ the number of channels that were not selected by any SU in time slot $t$. Since $M>N+1, L_{t} \geq 2$ for $t>0$. Therefore

$$
P\left(V_{t_{1}-1}^{i m}=2\right)=\left(\begin{array}{c}
L_{t_{1}-1} \\
2
\end{array}\right)(1-D C)^{2} D C^{L_{t_{1}-1}-2}>0,
$$

However, according to the strategy under imperfect monitoring, given $k$ static SUs before time $t_{1}$ and 2 channels are observed idle in time slot $t_{1}$, the remaining $N-k$ SUs randomly explore among the 2 channels, therefore,

$$
\begin{aligned}
& P\left(Y_{t_{1}}^{i m}=1 \mid S_{t_{1}-1}^{i m}=k, V_{t_{1}-1}^{i m}=2\right) \\
= & (N-k)(1-D C)\left(\frac{1}{2}\right)^{N-k-1} \\
> & 0
\end{aligned}
$$

which implies that :

$$
P\left(Y_{t_{1}}^{i m}=0 \mid S_{t_{1}-1}^{i m}=k, V_{t_{1}-1}^{i m}=2\right) \neq 1,
$$

for $k \in\{0,1, . . N-1\}$.

(8) and (10) together contradict (7).

\section{Simulation Results}

In this section, we analyze the dependency of the convergence time of designed strategies with respect to different environmental parameters. Each of the simulation results in this section is the average of 1000 independent simulations.

In our simulation we consider a cognitive radio system operating on $M=16$ channels. We analyze the convergence time of the two strategies for three duty cycle scenarios: the low PU activity level $(D C=0.3)$, shown in figure (2a), the medium PU activity level $(D C=0.5)$, shown in figure (2b) and the heavy PU activity level $(D C=0.7)$, shown in figure (2c). Under each scenario, we compare the convergence time for strategies under both perfect and imperfect monitoring scenarios with respect to the number of SUs within the system. Figure 3 shows the ratio of the convergence time under perfect monitoring to the convergence time under imperfect monitoring for three duty cycle levels.

First, we notice that the simulation results for the strategy under perfect monitoring accurately match the theoretical estimates of the convergence time, thus validating the theoretical analysis in Section V-A. Moreover, we can observe that the convergence time increases with $D C$ and number of SUs $N$ for both strategies. Finally, the results show the ratio of the convergence time between the strategy under imperfect scenario and the strategy under perfect monitoring is a decreasing function of $N$ and $D C$. In other words increased PU activity or SU competition amplify the loss caused by a less informative channel state. More specifically, under low PU activity $(D C=0.3)$, the difference between the convergence time of the two strategies is negligible even for relative large $N(N=11)$. However, when PU activity level is high $(D C=0.7)$, this ratio drops significantly as $N$ increases. Combining all these results, we conclude that the loss due to imperfect monitoring under i.i.d. homogeneous channels model is inevitable in the sense of longer convergence time. However, this loss is not significant under low PU activity levels and can be balanced by controlling the number of SUs operating in the system under high PU activity levels.

\section{CONCLUSION}

To our knowledge, this paper is the first to analyze the impact of imperfect information on the performance of autonomous secondary users' attempting to opportunistically exploit spectrum resources. The significance of imperfect monitoring lies in less requirements for signal detection techniques in real scenarios. The purpose of this paper is to addresses the question of how the quality of the information about past channel activity affects the SUs' long-term individual throughput.

We devised channel selection strategies for SUs under perfect monitoring and imperfect monitoring scenarios. Our 


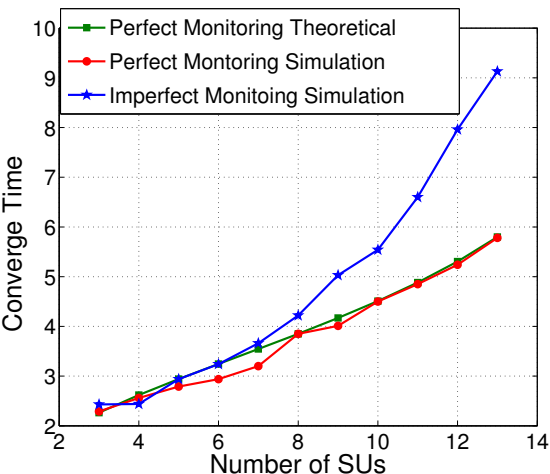

(a) $\mathrm{DC}=0.3$

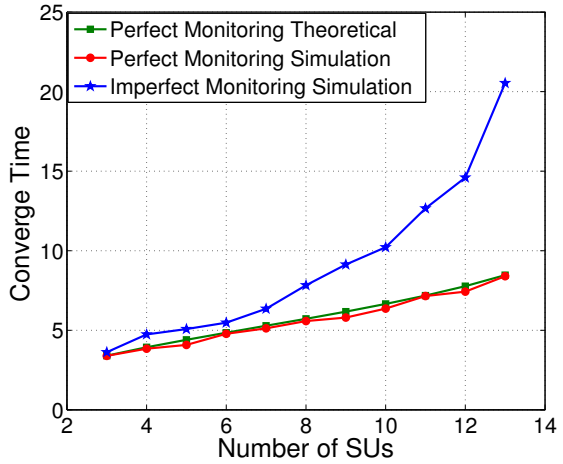

(b) $\mathrm{DC}=0.5$

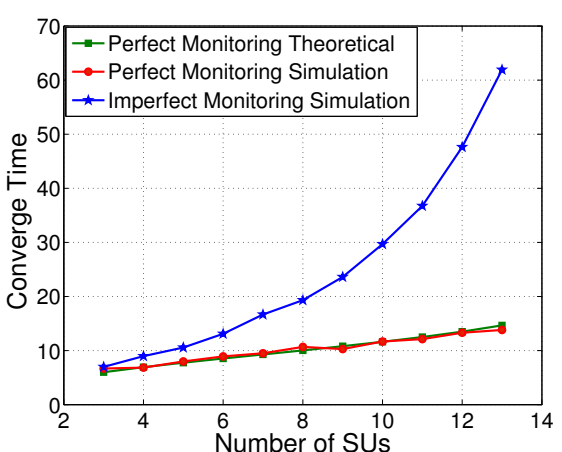

(c) $\mathrm{DC}=0.7$

Figure 2: $M=16$. Simulation result based on 1000 repeated experiments. The convergence time of strategies under perfect and imperfect monitoring depends on duty cycle $(D C)$ and number of SUs $(N)$.

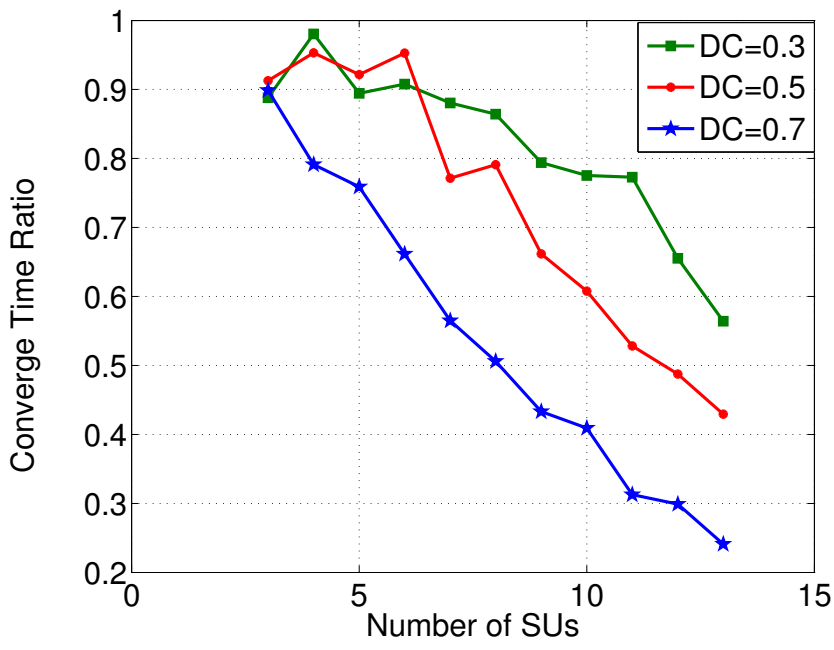

Figure 3: $M=16$. The ratio of the convergence time of strategy under the perfect monitoring scenario to the convergence time of the strategy under the imperfect monitoring scenario.

theoretical findings showed that both perfect and imperfect monitoring strategies converge to a static orthogonal allocation of the channels. The simulation results showed that under imperfect monitoring the ambiguity about the type of activity observed in the channels reduced the rate of convergence and the performance loss depended on the level of PU activity and on the amount of competition between SUs.

In this paper, we focused on homogeneous channels. We will consider the imperfect monitoring channel selection strategy design for non-homogenous channel occupancy in future research work

\section{REFERENCES}

[1] Y. Xu, A. Anpalagan, Q. Wu, L. Shen, Z. Gao, and J. Wang, "Decisiontheoretic distributed channel selection for opportunistic spectrum access: Strategies, challenges and solutions," Communications Surveys Tutorials, IEEE, vol. 15, pp. 1689-1713, April 2013.

[2] J. Zhao and X. Wang, "Channel sensing order in multi-user cognitive radio networks," in IEEE International Symposium on Dynamic Spectrum Access Networks (DYSPAN), pp. 397-407, 2012.
[3] Z. Khan, J. Lehtomäki, L. Dasilva, and M. Latva-aho, "Autonomous sensing order selection strategies exploiting channel access information," IEEE Transactions on Mobile Computing, vol. 12, no. 2, pp. 274-288, 2013.

[4] J. Unnikrishnan and V. V. Veeravalli, "Algorithms for Dynamic Spectrum Access With Learning for Cognitive Radio," IEEE Transactions on Signal Processing, vol. 58, no. 2, pp. 750-760, 2010.

[5] X. Y. Wang, A. Wong, and P.-H. Ho, "Dynamic markov-chain monte carlo channel negotiation for cognitive radio," in IEEE INFOCOM, pp. 1-5, 2010.

[6] Y. Gai, B. Krishnamachari, and R. Jain, "Learning multiuser channel allocations in cognitive radio networks: A combinatorial multi-armed bandit formulation," in IEEE Symposium on New Frontiers in Dynamic Spectrum (DYSPAN), pp. 1-9, 2010.

[7] C. Xin and X. Cao, "A cognitive radio network architecture without control channel," in IEEE GLOBECOM, pp. 1-6, 2009.

[8] B. Wang, Z. Ji, and K. Liu, "Self-learning repeated game framework for distributed primary-prioritized dynamic spectrum access," in 4th Annual IEEE Communications Society Conference on Sensor, Mesh and Ad Hoc Communications and Networks. SECON '07., pp. 631-638, 2007.

[9] Y. Wu, B. Wang, and K. Liu, "Repeated spectrum sharing game with self-enforcing truth-telling mechanism," in IEEE International Conference on Communications. ICC '08., pp. 3583-3587, 2008.

[10] Y. Xu, J. Wang, Q. Wu, A. Anpalagan, and Y.-D. Yao, "Opportunistic spectrum access in unknown dynamic environment: A game-theoretic stochastic learning solution," IEEE Transactions on Wireless Communications, vol. 11, no. 4, pp. 1380-1391, 2012.

[11] Y. Xiao and M. van der Schaar, "Dynamic spectrum sharing among repeatedly interacting selfish users with imperfect monitoring," IEEE Journal on Selected Areas in Communications, vol. 30, no. 10, pp. 1890 $1899,2012$.

[12] Y. Xiao and M. van der Schaar, "Distributed spectrum sharing policies for selfish users with imperfect monitoring ability," in Conference Record of the Forty Sixth Asilomar Conference on Signals, Systems and Computers (ASILOMAR), pp. 661-665, 2012.

[13] K. Liu, Q. Zhao, and B. Krishnamachari, "Decentralized multi-armed bandit with imperfect observations," in 48th Annual Allerton Conference on Communication, Control, and Computing (Allerton), pp. 1669-1674, IEEE, 2010.

[14] K. Liu, Q. Zhao, and B. Krishnamachari, "Distributed learning under imperfect sensing in cognitive radio networks," in Conference Record of the Forty Fourth Asilomar Conference on Signals, Systems and Computers (ASILOMAR), pp. 671-675, 2010.

[15] Y. Chen, Q. Zhao, and A. Swami, "Joint design and separation principle for opportunistic spectrum access in the presence of sensing errors," IEEE Transactions on Information Theory, vol. 54, no. 5, pp. $2053-$ 2071, 2008.

[16] J. L. Kemeny, John G.; Snell, Finite Markov Chains. ISBN 978-0-38790192-3, Springer, 2 ed., 1960

[17] C. M. Grinstead and J. L. Snell., Introduction to Probability. American Mathematical Society, 2 ed., 1997. 